\title{
Using Physical Models to Improve Thermal IR Detection of Buried Mines
}

\author{
D.-H. Chen, I. K. Sendur, W.-J. Liao and B. A. Baertlein* \\ The Ohio State University ElectroScience Laboratory \\ 1320 Kinnear Road, Columbus, OH 43212
}

\begin{abstract}
Many aspects of a buried mine's thermal IR signature can be predicted through physical models, and insight provided by such models can lead to better detection. Several techniques for exploiting this information are described. The first approach involves ML estimation of model parameters and followed by classification of those parameters. We show that this approach is related to an approximate evaluation of an integral over the parameters that arises in a Bayesian formulation. This technique is compared with a generalized likelihood ratio test (GLRT) and with computationally efficient, model-free approaches, in which soil temperature data are classified directly. The benefit of using the temporal information is also investigated. Algorithm performance is illustrated using broadband IR imagery of buried mines acquired over a 24 hour period. It is found that the detection performance at a suitably selected time is comparable to the performance achieved by processing all times. The performance of the GLRT, for which detection is based only on the residual error, is inferior to a classifier using the parameters.
\end{abstract}

Keywords: IR, thermal, buried mines, modeling, space-time processing

\section{INTRODUCTION}

The thermal IR signature of a buried land mine depends on a number of mine-related or environmental parameters. This dependence is poorly understood, which limits one's ability to predict the signature and to design effective mine detection algorithms.

Recent studies of the physics that govern thermal IR signatures of buried land mines have shed light on the phenomena at work in mine signatures. A simulation tool described by Sendur and Baertlein ${ }^{1}$ has shown an ability to predict these signatures. Recent work in this area appears in a companion paper. ${ }^{2}$ Physical signature models are complex, but an analysis of measured signature data and use of the aforementioned tool suggest that relatively simple models can be constructed for these signatures. We show herein that key model parameters can be estimated from imagery, and that such simple models can be used to improve buried mine detection.

Several methods can be used to exploit information derived through modeling. A Bayesian approach to detection leads to an integral over unknown model parameters. Alternatively, a generalized likelihood ratio test (GLRT) can be used, in which detection is based on the residual error between data and model having used ML estimates of the model parameters. We describe another approach, referred to herein as the "estimator-classifier" technique, which makes explicit use of the model parameters. We show that the estimator-classifier technique is consistent with an approximate evaluation of the Bayesian parameter integrals.

Mine signatures are time varying, and we also investigate the benefits of using both spatial and temporal information in detection. Temporal signature information is not available in many mine-detection operations, but it is attractive for humanitarian demining, in which the observer is able to acquire multiple looks at the region of interest. Although it has long been known that mine signatures are time dependent, this fact has seldom been exploited for detection. We have identified only one group ${ }^{3}$ that has previously explored this concept.

The performance of several detection algorithms is evaluated using broadband IR data acquired at regular intervals over a 24 hour cycle. The estimator-classifier technique is compared with the GLRT, and the benefit of basing detection on single versus multiple time samples is determined. All of these methods are computationally expensive. For comparison, we also explore some computationally efficient model-free methods, including a classifier of temporal signature behavior and the well-known "RX" algorithm of Reed and Yu. ${ }^{4}$

Corresponding author: B.A.B. (614) 292-0076 (voice), (614) 292-7297 (fax), baertlein.1@osu.edu 
This work is organized in four major sections. The problem of mine detection using a space-time model is formulated in Section 2. Our choice of model is motivated by some aspects of the data, which we describe in Section 3. An example multi-temporal data set of thermal IR signatures is also discussed in that section. A computationally efficient (albeit less effective) model-free algorithm is described in Section 4, which involves a classifier trained to recognize mines from their thermal history. Detection results in the form of ROC curves are reported in Section 5. Concluding remarks appear in Section 6.

\section{SPACE-TIME DETECTION ALGORITHM}

We can formulate a model-based detection algorithm for the spatial and temporal characteristics of the signature as follows: The measured image data $\mathbf{D}(t)$ are assumed to comprise clutter or the mine signature in additive noise $\mathbf{N}(t)$, for which we write

$$
\mathbf{D}(t)=\left\{\begin{array}{ll}
\mathbf{G}_{0}(\Theta, t)+\mathbf{N}(t), & H_{0} \\
\mathbf{G}_{1}(\boldsymbol{\Theta}, t)+\mathbf{N}(t), & H_{1}
\end{array} \quad t=t_{1}, t_{2}, \ldots\right.
$$

In what follows we suppress the time dependence to simplify the notation. Here $\mathbf{G}_{k}(\boldsymbol{\Theta})$ is the (assumed known) physical model (described below) for the signal under hypothesis $H_{k}, k=0,1$, given model parameters $\Theta$. The noise $\mathbf{N}$ is assumed to be independent of the hypothesis and to have the distribution $\mathbf{N} \sim f_{\mathbf{N}}$. Adopting a Bayesian approach, the distribution of the parameters $\Theta \sim f_{\Theta \mid H_{k}}(\Theta)$ will depend on the hypothesis, and we have

$$
f_{\mathbf{D} \mid H_{k}}(\mathbf{D})=\int d \boldsymbol{\Theta}_{k} f_{\boldsymbol{\Theta}_{k} \mid H_{k}}\left(\boldsymbol{\Theta}_{k}\right) f_{\mathbf{N}}\left(\mathbf{D}-\mathbf{G}_{k}\left(\boldsymbol{\Theta}_{k}\right)\right)
$$

which leads to the likelihood ratio

$$
\Lambda(\mathbf{D})=\frac{f_{\mathbf{D} \mid H_{1}}(\mathbf{D})}{f_{\mathbf{D} \mid H_{0}}(\mathbf{D})}=\frac{\int d \boldsymbol{\Theta} f_{\mathbf{N}}\left(\mathbf{D}-\mathbf{G}_{1}(\boldsymbol{\Theta})\right) f_{\boldsymbol{\Theta} \mid H_{1}}(\boldsymbol{\Theta})}{\int d \boldsymbol{\Theta} f_{\mathbf{N}}\left(\mathbf{D}-\mathbf{G}_{0}(\boldsymbol{\Theta})\right) f_{\boldsymbol{\Theta} \mid H_{0}}(\boldsymbol{\Theta})}
$$

Direct evaluation of this expression is possible if the densities $f_{\mathbf{N}}$ and $f_{\Theta \mid H_{k}}$ are available. This approach has been used previously by Gao and Collins ${ }^{5}$ for the GEM-3 EMI sensor. EMI sensors are largely independent of the environment and, hence, only aspects of the target need be modeled. In their work, Gao and Collins modeled mine parameters such as tilt, depth, metal content and metal distribution. In principle, the same approach could be used here: measurements of relevant environmental parameters could be used with the signature model to predict the observed signature as a function of unknown variables including the mine depth and size. Our experience to date with the model suggests that such an approach would not be successful. Although the spatial shape of a mine signature can be predicted with some confidence, the temperature contrast over the mine is strongly dependent on environmental conditions including the soil emissivity, soil moisture distribution with depth and the initial temperature distribution of the soil. Measurements of those data are often not available, which makes it difficult to accurately predict contrast. In addition, the thermal signature model is computationally expensive to run, and it would be prohibitively time consuming to synthesize the signatures necessary to evaluate Eq. (3) directly.

A common alternative approach to similar problems is the GLRT, ${ }^{6}$ in which a maximum likelihood estimate $\hat{\boldsymbol{\Theta}}$ of the model parameters is used as follows:

$$
\Lambda_{G}(\mathbf{D})=\frac{\max _{\boldsymbol{\Theta}} f_{\mathbf{D} \mid \Theta, H_{1}}}{\max _{\boldsymbol{\Theta}} f_{\mathbf{D} \mid \Theta, H_{0}}}=\frac{f_{\mathbf{N}}\left(\mathbf{D}-\mathbf{G}_{1}\left(\tilde{\boldsymbol{\Theta}}_{1}\right)\right)}{f_{\mathbf{N}}\left(\mathbf{D}-\mathbf{G}_{0}\left(\tilde{\boldsymbol{\Theta}}_{0}\right)\right)}
$$

where the estimated parameters $\tilde{\Theta}_{k}$ maximize the densities $f_{\mathbf{D} \mid \Theta, H_{k}}$. Thus, GLRT detection is based primarily on the density of the model error. The model parameters are considered indirectly, via their contribution to the error. The estimator-correlator method ${ }^{6}$ uses a similar approach, in which the parameters are estimated and the resulting model signature is correlated with the data. For Gaussian statistics, detection is based on the correlation.

In this work we propose a somewhat different approach. We estimate parameters $\hat{\boldsymbol{\Theta}}_{k}$ which minimize the residual error $\left|\mathbf{D}-\mathbf{G}_{k}\left(\hat{\boldsymbol{\Theta}}_{k}\right)\right|$ and supply these values to a classifier. We refer to this approach as an estimator-classifier, by analogy with the estimator-correlator technique. The technique is motivated by an approximate evaluation of the 
Bayesian representation in Eq. (3). Consider the case in which the model $\mathbf{G}_{k}$ is a good fit to the data $\mathbf{D}$ for the parameter value $\hat{\boldsymbol{\Theta}}_{k}$. In that case, $f_{\mathbf{N}}\left(\mathbf{D}-\mathbf{G}\left(\boldsymbol{\Theta}_{k}\right)\right)$ will have a well defined maximum for $\boldsymbol{\Theta}_{k}=\hat{\boldsymbol{\Theta}}_{k}$ and we can write

$$
f_{\mathbf{D} \mid H_{k}}(\mathbf{D}) \approx f_{\boldsymbol{\Theta}_{k} \mid H_{k}}\left(\hat{\boldsymbol{\Theta}}_{k}\right) \int d \boldsymbol{\Theta}_{k} f_{\mathbf{N}}\left(\mathbf{D}-\mathbf{G}_{k}\left(\boldsymbol{\Theta}_{k}\right)\right) \approx E_{k} f_{\boldsymbol{\Theta}_{k} \mid H_{k}}\left(\hat{\boldsymbol{\Theta}}_{k}\right) f_{\mathbf{N}}\left(\mathbf{D}-\mathbf{G}_{k}\left(\hat{\boldsymbol{\Theta}}_{k}\right)\right)
$$

where we have assumed that $f_{\boldsymbol{\Theta}_{k} \mid H_{k}}$ is smooth, and the constant factor

$$
\begin{aligned}
E_{k} & =\int d \boldsymbol{\Theta}_{k} h_{k}\left(\boldsymbol{\Theta}_{k}\right) \\
h_{k}(\boldsymbol{\Theta}) & =\frac{f_{\mathbf{N}}\left(\mathbf{D}-\mathbf{G}_{k}(\boldsymbol{\Theta})\right)}{f_{\mathbf{N}}\left(\mathbf{D}-\mathbf{G}_{k}\left(\hat{\boldsymbol{\Theta}}_{k}\right)\right)}
\end{aligned}
$$

is related to the residual error. The function $h_{k}$ satisfies $h_{k}\left(\hat{\boldsymbol{\Theta}}_{k}\right)=1$ and $(\partial / \partial \boldsymbol{\Theta}) h_{k}\left(\hat{\boldsymbol{\Theta}}_{k}\right)=0$. Defining the matrix

$$
\left[\boldsymbol{\Omega}_{k}\right]_{i j}=-\left.\frac{\partial}{\partial \Theta_{i}} \frac{\partial}{\partial \Theta_{j}} h_{k}\left(\Theta_{k}\right)\right|_{\hat{\Theta}_{k}}
$$

We must also have $\left(\boldsymbol{\Theta}-\hat{\boldsymbol{\Theta}}_{k}\right)^{T} \boldsymbol{\Omega}_{k}\left(\boldsymbol{\Theta}-\hat{\boldsymbol{\Theta}}_{k}\right)>>1$ for a well defined maximum in $f_{\mathbf{N}}$, which implies that near $\hat{\boldsymbol{\Theta}}_{k}$ we may use the approximation

$$
h_{k}(\boldsymbol{\Theta}) \approx \exp \left(-\left(\boldsymbol{\Theta}-\hat{\boldsymbol{\Theta}}_{k}\right)^{T} \boldsymbol{\Omega}_{k}\left(\boldsymbol{\Theta}-\hat{\boldsymbol{\Theta}}_{k}\right) / 2\right)
$$

This result is distribution free but immediately evident for normally distributed $\mathbf{N}$. We are led to the result

$$
E_{k} \propto \operatorname{det}\left(\Omega_{k}\right)^{1 / 2}
$$

Thus,

$$
\Lambda(\mathbf{D}) \approx \frac{f_{\boldsymbol{\Theta} \mid H_{1}}\left(\hat{\boldsymbol{\Theta}}_{1}\right) \int d \boldsymbol{\Theta} f_{\mathbf{N}}\left(\mathbf{D}-\mathbf{G}_{1}(\boldsymbol{\Theta})\right)}{f_{\boldsymbol{\Theta} \mid H_{0}}\left(\hat{\boldsymbol{\Theta}}_{0}\right) \int d \boldsymbol{\Theta} f_{\mathbf{N}}\left(\mathbf{D}-\mathbf{G}_{0}(\boldsymbol{\Theta})\right)}=\frac{f_{\boldsymbol{\Theta} \mid H_{1}}\left(\hat{\boldsymbol{\Theta}}_{1}\right)}{f_{\boldsymbol{\Theta} \mid H_{0}}\left(\hat{\boldsymbol{\Theta}}_{1}\right)} \frac{f_{\mathbf{N}}\left(\mathbf{D}-\mathbf{G}_{1}\left(\hat{\boldsymbol{\Theta}}_{1}\right)\right)}{f_{\mathbf{N}}\left(\mathbf{D}-\mathbf{G}_{0}\left(\hat{\boldsymbol{\Theta}}_{0}\right)\right)} \frac{\operatorname{det}\left(\boldsymbol{\Omega}_{1}\right)^{1 / 2}}{\operatorname{det}\left(\boldsymbol{\Omega}_{0}\right)^{1 / 2}}
$$

It is notable that a GLRT approach in which the covariance matrix of the imagery is estimated using an ML approach yields a likelihood ratio that involves only the ratio of covariance matrix determinants. ${ }^{4}$ In the estimator-classifier formulation detection is performed on the basis of the class-conditioned densities of $\Theta$ and the residual model error $\left|\mathbf{D}-\mathbf{G}_{k}\left(\hat{\boldsymbol{\Theta}}_{k}\right)\right|$. Since a classifier of $\hat{\boldsymbol{\Theta}}_{k}$ estimates quantities comparable to $f_{\boldsymbol{\Theta} \mid H_{k}}\left(\hat{\boldsymbol{\Theta}}_{k}\right)$, it is reasonable to use such classifiers, with additional information on the residual error, for detection. This is the basis for the estimator-classifier approach. In our formulation, a nonlinear optimization procedure is used to find $\hat{\boldsymbol{\Theta}}_{k}$, and the corresponding model error. We use a support vector machine $(\mathrm{SVM})^{7,8}$ as a classifier. Radial basis functions are used, and the basis function parameter is adjusted to produce the best performance in leave-one-out testing of the results.

It is also easy to show that an expression resembling the GLRT can be derived from Eq. (2) using this approach if one makes the approximation that the densities $f_{\boldsymbol{\Theta} \mid h_{k}}$ are strongly peaked about parameter values $\tilde{\boldsymbol{\Theta}}_{k}$. A lack of a priori information regarding the parameters motivated our investigation of the estimator-classifier technique.

\section{SIGNATURE MODEL DEVELOPMENT}

We now define signature models $\mathbf{G}_{k}(\boldsymbol{\Theta})$ to be used for targets and clutter. IR signatures of buried land mines are functions of both space and time. We describe the spatial and temporal characteristics of these signatures using a combination of experimental data and modeling.

\subsection{A Measured Data Set}

In this work we use signatures measured as part of the 1998 NVESD MWIR and LWIR diurnal collection study. During the summer of 1998 a staring IR camera was used to image surface-laid and buried targets at two sites, Fort A.P. Hill, VA and Fort Huachuca, AZ. The layout of the minefields were similar for these two collections, and a description of the Ft. A.P. Hill data set is shown in Fig. 1. Data were acquired at 15 minute intervals for a duration of roughly 24 hours. Both Agema $570(8-12 \mu \mathrm{m})$ and Amber Radiance $(3-5 \mu \mathrm{m})$ cameras were used. We used data from the Agema camera, one image of which is shown in Fig. 2. These data were collected 1 July 1998 at Ft. A. P. Hill (Collection 3). Meteorological data were collected with the imagery. For this study fifteen target chips (one for each buried mine) and an equal number of clutter chips were extracted from this imagery. Examples of those chips are shown in Fig. 3. 

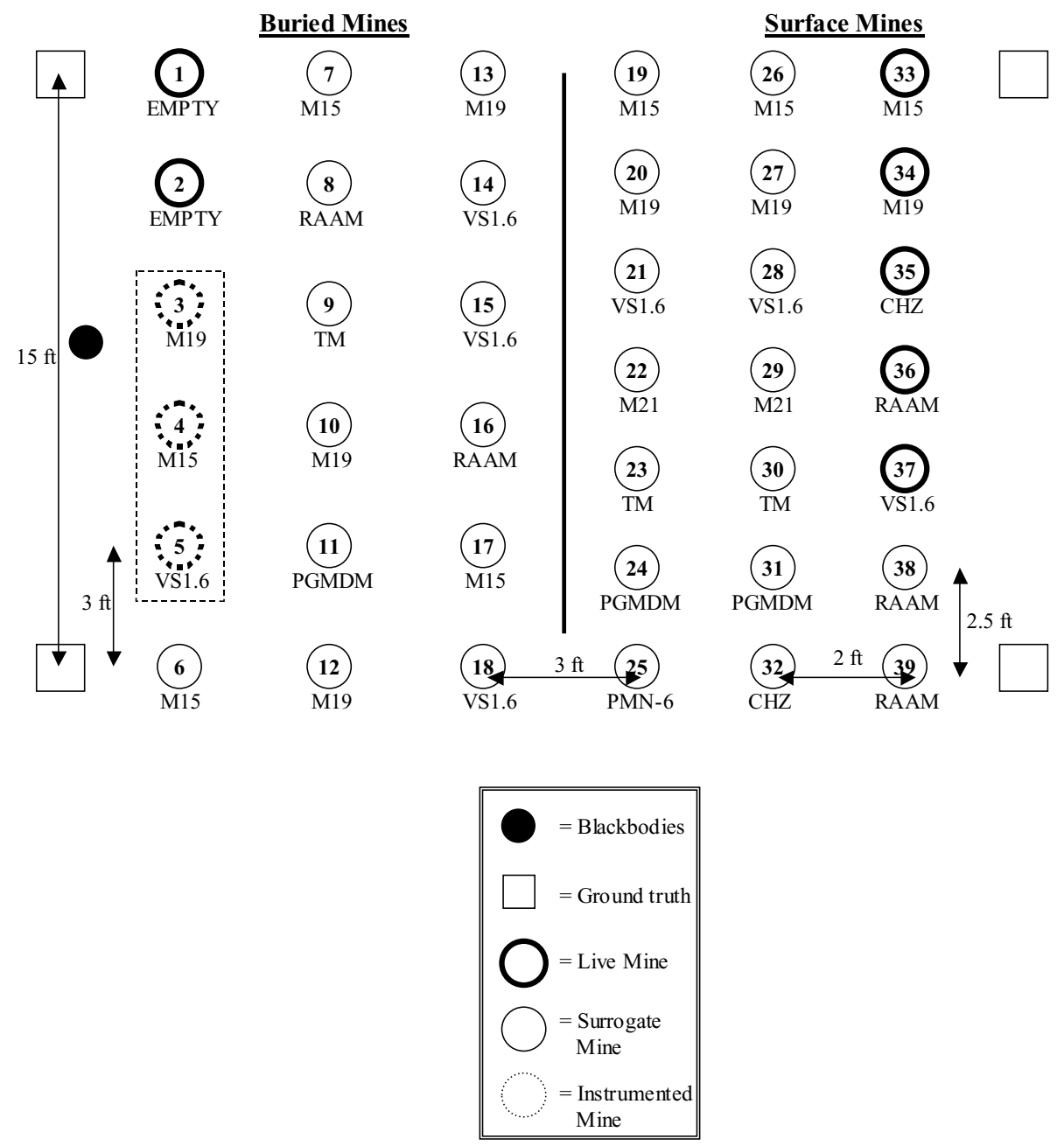

Figure 1. Mine layout for the AP Hill collection. 


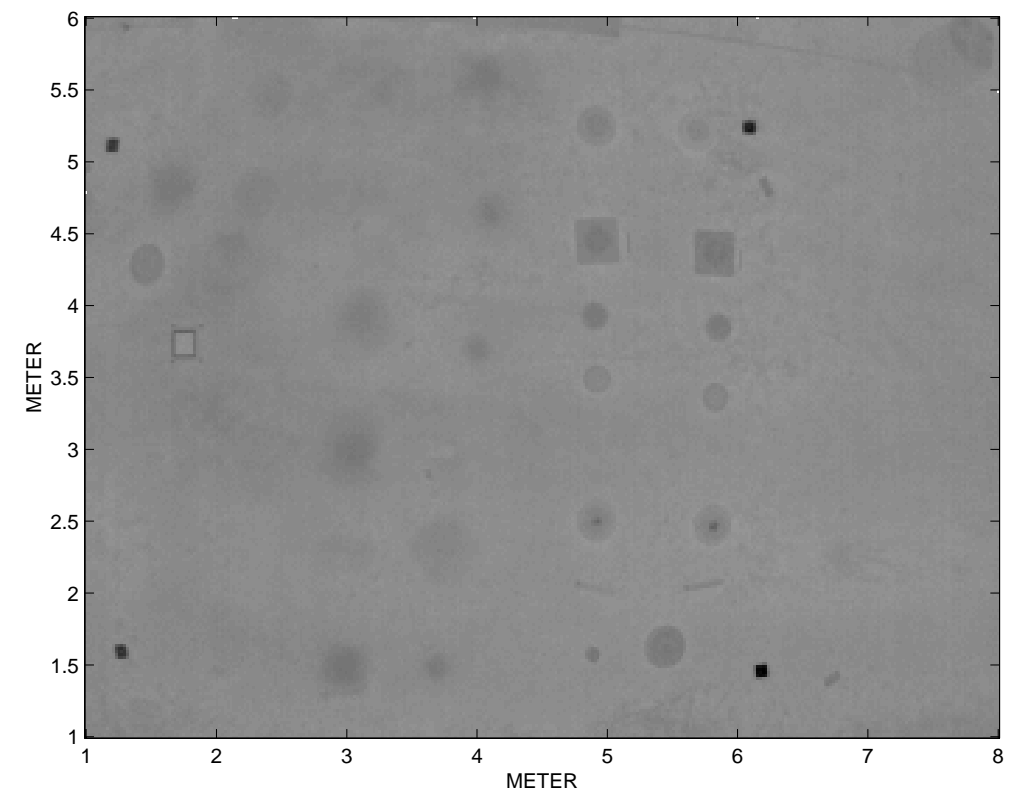

Figure 2. Example imagery from the Fort A. P. Hill collection.

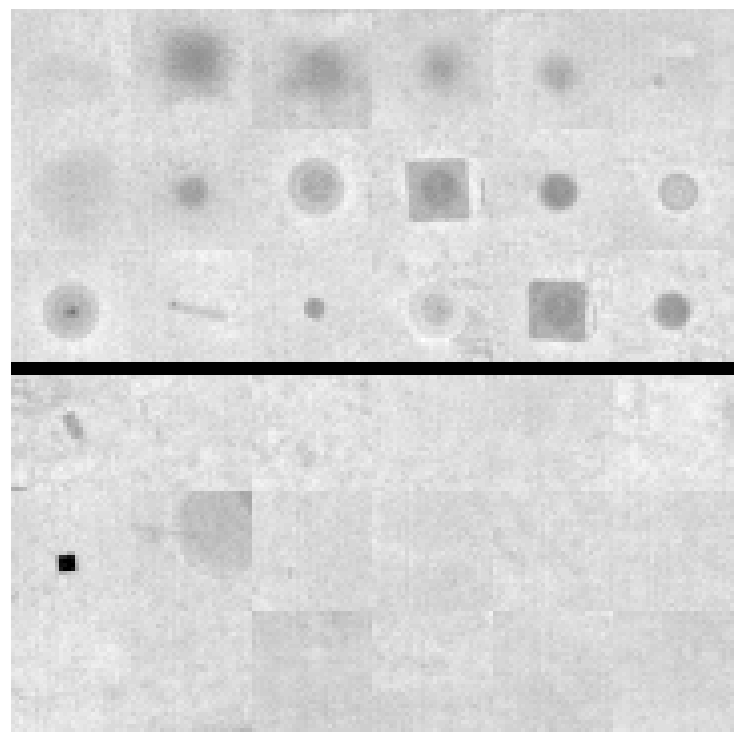

Figure 3. Chips from buried mines (above) and clutter (below). 


\subsection{Characteristics of Thermal IR Buried Mine Signatures}

We can broadly characterize the signature model parameters as mine-related or environmentally related. The role of environmental variables is discussed in a companion paper. ${ }^{2}$ In that work it is shown that the signature's shape is weakly dependent on the environmental variables. In addition, many details of the mine's construction have a small effect on soil surface temperature, since diffusion of the thermal signature through the overlying soil tends to smooth small-scale spatial variations. It remains to consider the signature's temporal behavior and the relation of mine-related variables to signature shape.

Consider first the dependence of the signature shape on mine-related variables such as the mine diameter and burial depth. Each buried mine comprises a single sample of a specific mine diameter and burial depth. Since the number of measured mine signatures is small (only 15), it is appropriate to examine the influence of these variables using modeling. Simulations of surface temperature contrast as a function of time and depth reveal that the spatial character of the signature is (after normalization to unit amplitude) only weakly dependent on mine depth. Although the peak amplitude of the contrast changes from positive to negative over the course of a diurnal cycle, the spatial distribution of the signature varies only moderately. Examples of this behavior are shown in Fig. 4 where we find that the shape of the signature is largely invariant with time. Fig. 5 shows that the depth of the mine also has little effect on the shape of the signature. The mine diameter has a more significant effect on the shape of the signature, as shown in Fig. 6. We see that for a fixed time and burial depth, the radius of the signature varies strongly with mine diameter. This suggests that the signature shape can be modeled with a small number of parameters, one of which should be related to diameter. These findings also suggest that the spatial dependence of the signature can be decoupled from the temporal dependence.

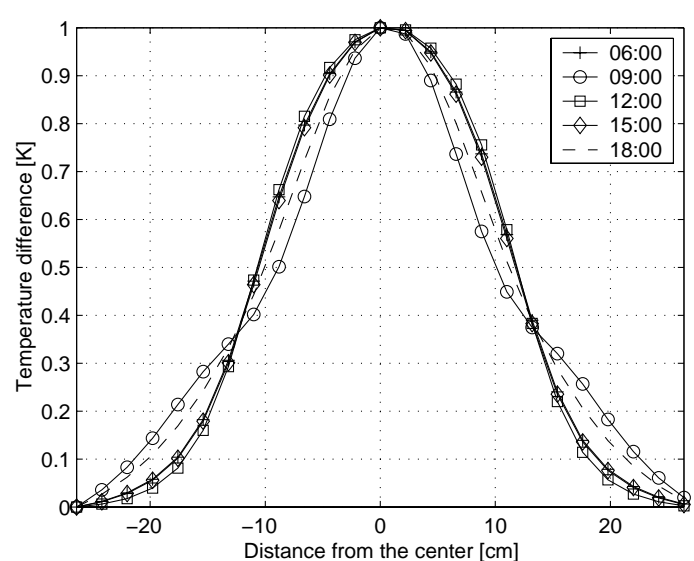

(a) $3.32 \mathrm{~cm} \mathrm{depth}$

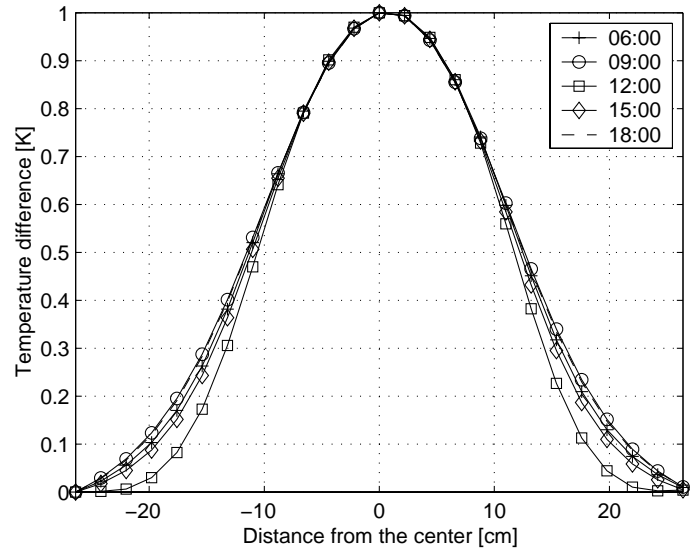

(b) $6.64 \mathrm{~cm} \mathrm{depth}$

Figure 4. Spatial distribution of temperature contrast for mines buried at two depths and various times. The curves have been normalized to produce unit maximum.

Conversely, the signature's temporal behavior is strongly dependent on the environment and, in particular, on the solar insolation. The effect is most noticeable during daylight hours when broken clouds are present, since the temperature of the thin layer of soil at the surface can change rapidly in response to the incident sunlight. Figure 7 presents temperature histories for buried mines in relation to clutter. The temperature history during daylight hours is strongly dependent on the incident solar flux, which varies as a result of solar insolation.

Although the temperature over a mine varies strongly with environmental conditions, detection is typically based on the thermal contrast - the difference between the temperature over a mine and the temperature of the surrounding soil. Plots the thermal contrast for buried mines are shown in Fig. 8. The curves pass through zero twice daily, and these times are referred to as "crossover times". For times in the early morning and late evening (i.e., between the thermal crossover times), a detectable contrast is present. The soil over a mine tends to be somewhat cooler during the evening hours, as predicted by the model. ${ }^{2}$ The model also predicts that the soil over a mine will be somewhat 


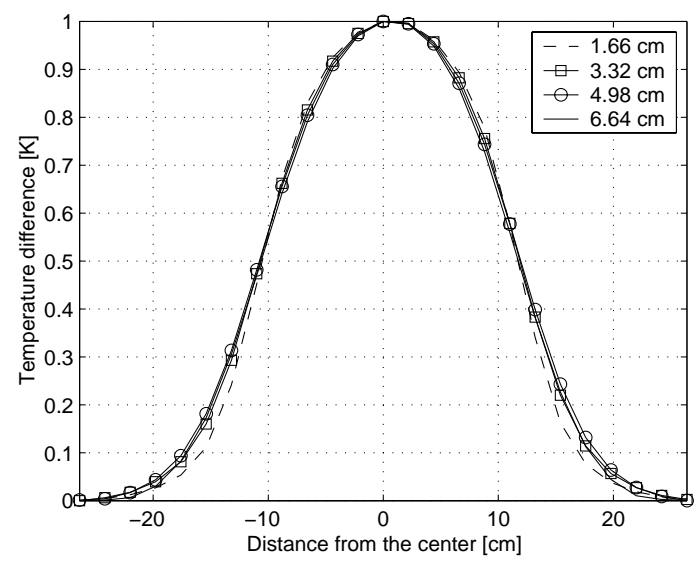

(a) 1200 Hours

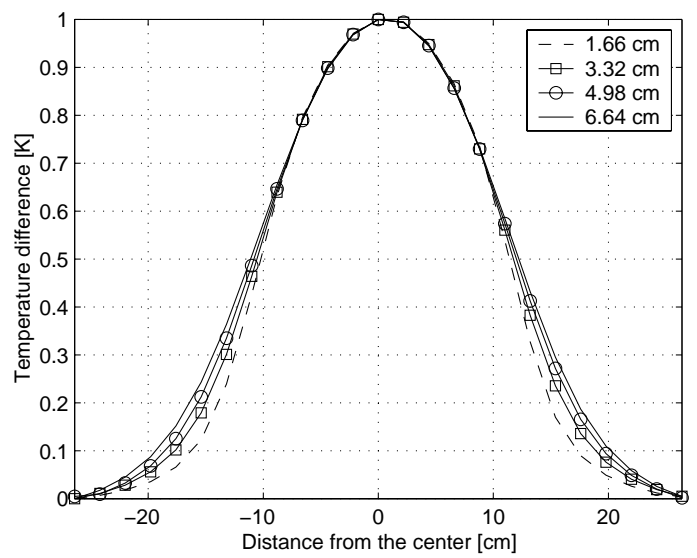

(b) 1500 Hours

Figure 5. Spatial distribution of temperature contrast for two times and various mine depths. The curves have been normalized to produce unit maximum.

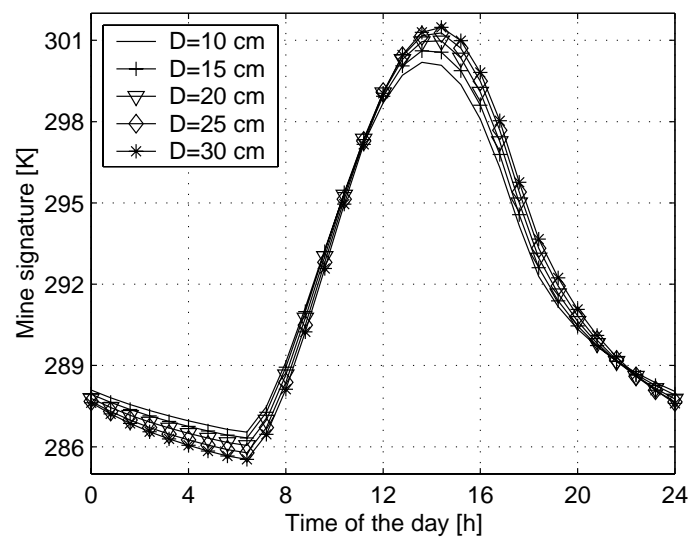

(a) Temporal variations in the temperature over the center of the mine.

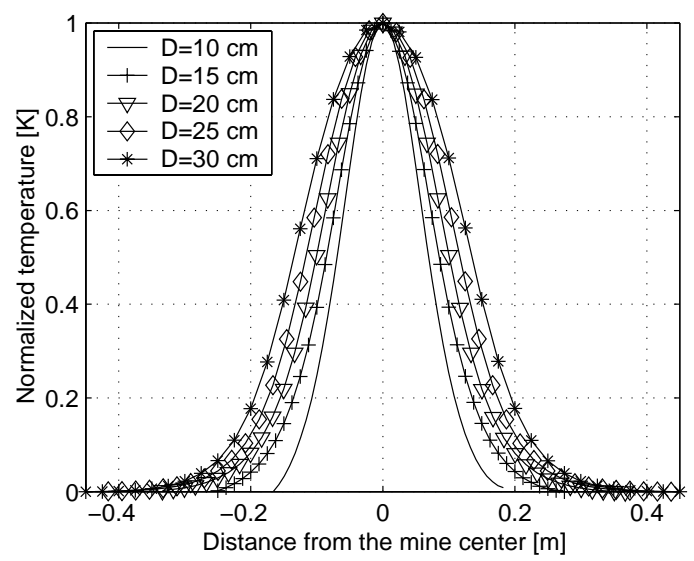

(b) Spatial distribution of temperature contrast for a fixed time. The curves have been normalized to unit maximum.

Figure 6. Variations in the signature with mine radius.

warmer during the day. The temperature difference between two points in the clutter chips are also shown in Fig. 8, where we see that those signatures are generally flat. One clutter chip contains a surface object, the temperature of which closely tracks the incident solar radiance.

A review of the results presented in this section suggests that spatial target signatures can be described by a model with a small number of physical parameters. Moreover, several of the physical parameters have a similar effect on the model, which implies that to first order the signature can be described by a smaller number of (possibly non-physical) parameters. Conversely, the temporal dependence of the signature is difficult to model, because it depends strongly on environmental factors. 


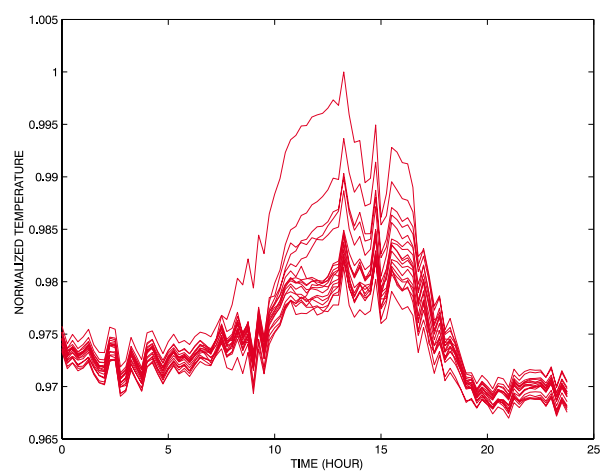

(a) Mines

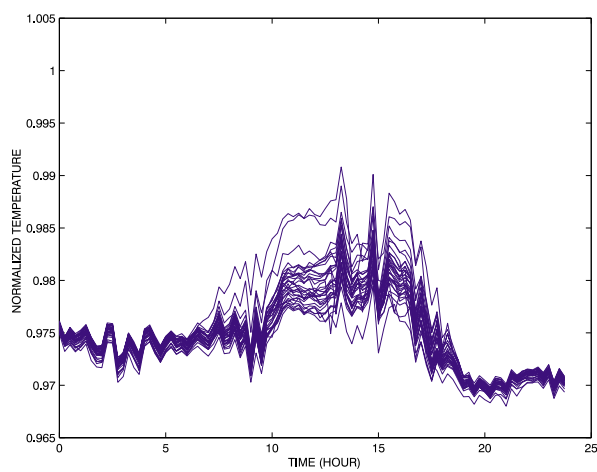

(b) Clutter

Figure 7. Temperature histories over buried mines and clutter

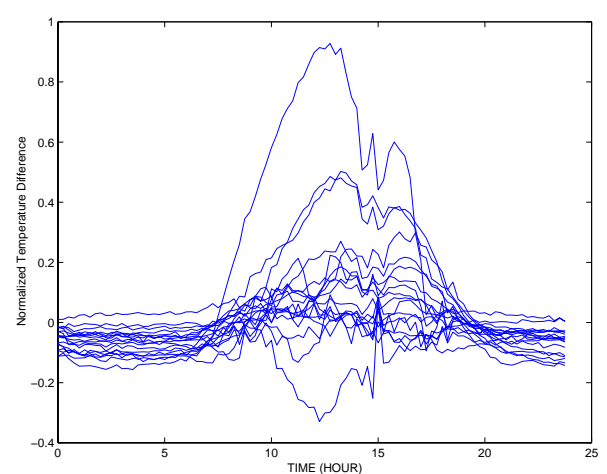

(a) Mines

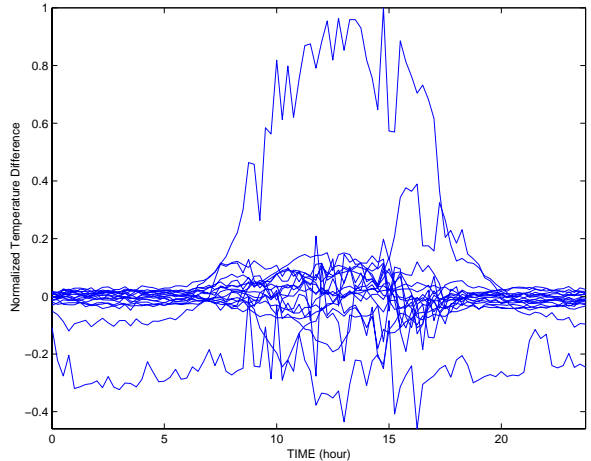

(b) Clutter

Figure 8. Thermal contrast over buried mines and clutter.

\subsection{Model Definition}

Since the shape and time dependence of the signature are only weakly coupled, and since the shape depends on a small number of parameters, it is attractive to represent the IR signature by a relatively simple parameteric model. The spatial signature dependence is particularly amenable to this treatment.

A good fit to the aforementioned data set can be obtained with the following simple expressions:

$$
\mathbf{G}\left(\Theta_{1}\right)=b(t)+\frac{c(t)}{1+(|\mathbf{r}| / \Delta)^{p}}
$$

in which

$$
\boldsymbol{\Theta}_{1}=\left[\begin{array}{llll}
b(t) & c(t) & \Delta & p
\end{array}\right]
$$

where $b(t)$ and $c(t)$ are the time-dependent background and contrast temperatures, $\Delta$ is a spatial scale factor, and $p$ controls the spatial decay rate of the signature. The same model is used for both clutter and mines. The quantities $\Delta$ and $p$ control the shape of the signature, and they subsume variations with mine diameter and depth. The time 
dependence is described by $b(t)$ and $c(t)$, of which only $c(t)$ is relevant to detection. The background temperature $b(t)$ can be obtained from modeling or measurements of the local environment.

As noted above, the mine contrast $c(t)$ and the background $b(t)$ depend strongly on a number of time-dependent quantities including the local solar insolation. A study of the experimental data shows that the background and contrast information are well approximated by a normalized reference signature $b_{0}(t)$ and $c_{0}(t)$ with random constants $b$ and $c$, which leads to

$$
\mathbf{G}(b, c, \Delta, p)=b b_{0}(t)+\frac{c c_{0}(t)}{1+(|\mathbf{r}| / \Delta)^{p}}
$$

where $b_{0}(t)$ and $c_{0}(t)$ are developed from models or from training data. An example of this model and it's fit to experimental data are shown in Fig. 9. Although the data are noisy, the model fits reasonably well.

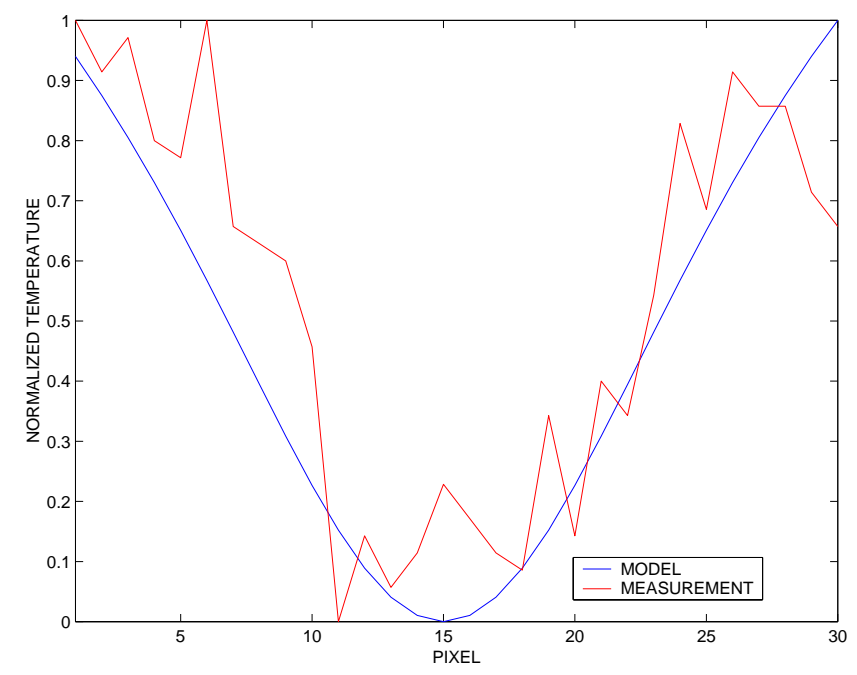

Figure 9. A comparison of the empirical model in Eq. (14) and measured data D.

\section{TEMPORAL DETECTION ALGORITHM}

Because the model $\mathbf{G}(\boldsymbol{\Theta})$ depends nonlinearly on the parameters $\Delta$ and $p$, a nonlinear optimization procedure must be used to find the ML estimates $\hat{\boldsymbol{\Theta}}_{k}$. This process is computationally expensive, which motivates consideration of other more efficient detection methods. Since the nonlinearities arise in the spatial signature dependence, one approach is to base detection entirely on the temporal signatures. It is relatively easy to adapt the foregoing approach to temporal-only processing.

Mine detection in multi-temporal imagery was investigated using two techniques. In the first approach we searched for spatial temperature anomalies. The time histories were sampled at six points taken roughly four hours apart starting at approximately 4:00 AM. This set of data was used with the well-known RX algorithm of Reed and $\mathrm{Yu}^{4}$ That techniques comprises a CFAR implementation of a GLRT detector for a known target shape with unknown spectral amplitude and covariance in spatially white Gaussian noise. An ML technique is used to solve for the unknown amplitude $c$. The algorithm produces a likelihood ratio derived from local estimates of the residual model error and clutter variance. Because RX attempts to match to a known target shape, some spatial signature information is used. Like the GLRT approach described above, the unknown amplitude $c$ is only used indirectly in detection, i.e., via its influence on the covariance matrix.

The second approach was model-free. We used a classifier to detect mines based on their temperature history. A support vector machine was trained on the image chips and used in classification. No spatial information was used in this detector. Each pixel in a chip was presented to the classifier, resulting in a binary distribution of single-pixel 
detections. That binary image was processed to reject small pixel groupings, and detection was based on the presence of a "blob" at the location of the target.

\section{RESULTS}

Figure 10 summarizes the performance of the estimator-classifier detector and the GLRT detector, both of which exploit the full space-time signatures. The figure also shows the performance of the model-free temporal-only detector using the SVM. The results used leave-one-out testing of the 30 image chips described in Section 3.1.

The improved performance of the estimator-classifier using space-time information suggests that there is significant detection information in the model parameters. The GLRT, which ignores that information, shows markedly worse performance. The performance of the temporal-only SVM classifier is comparable to that of the GLRT. The explicit use of spatial information, conveyed using the model parameters, appears to be correlated with good performance.

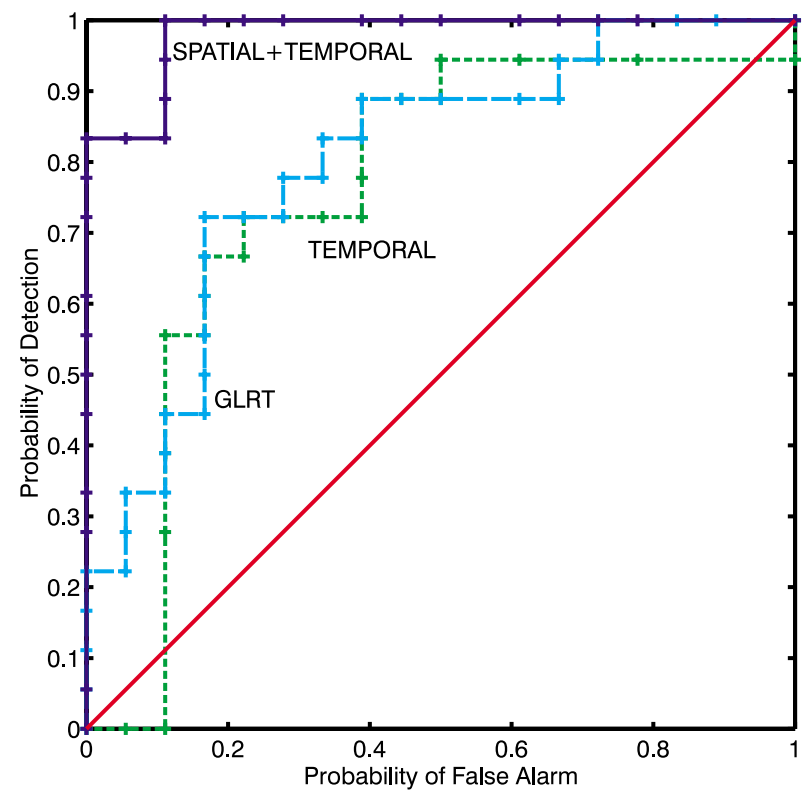

Figure 10. ROC curves for classifiers using (1) the estimator-classifier for the full space-time signature ("Spatial+Temporal"), (2) the GLRT for the space-time signature ("GLRT"), and (3) the SVM classifier using only temporal information ("Temporal").

Several of the algorithms discussed above use both spatial and temporal information in detection. Since the temporal information is often inconvenient or impossible to acquire, we next investigate its contribution to detection performance. This issues is addressed in Fig. 11 where we show detection of buried mines using a single "snap-shot" image and the entire time history. Two snap-shots are considered in Fig. 11. In the first, which occurred between crossover times, we see that the single-time and full-time performance are similar. The performance for the second snap-shot is dramatically worse. In that case, the sample time corresponds to a thermal crossover, when the mine signature is weak. We conclude that the performance of a detector that uses a single look can be comparable to that of a detector using the full time history but, as expected, performance is strongly dependent on the sample time and its relation to thermal crossover.

Finally we compare the performance of the relatively efficient detectors considered in Section 4 . The ROC curves generated by RX (with spatial and temporal information) and by leave-one-out testing of the SVM using temporal data only are shown in Fig. 12 for buried mines. We see that the classifier approach performs significantly better than the RX algorithm for these data. The results suggest that assuming an incorrect signature shape can be worse than ignoring the spatial information entirely. 


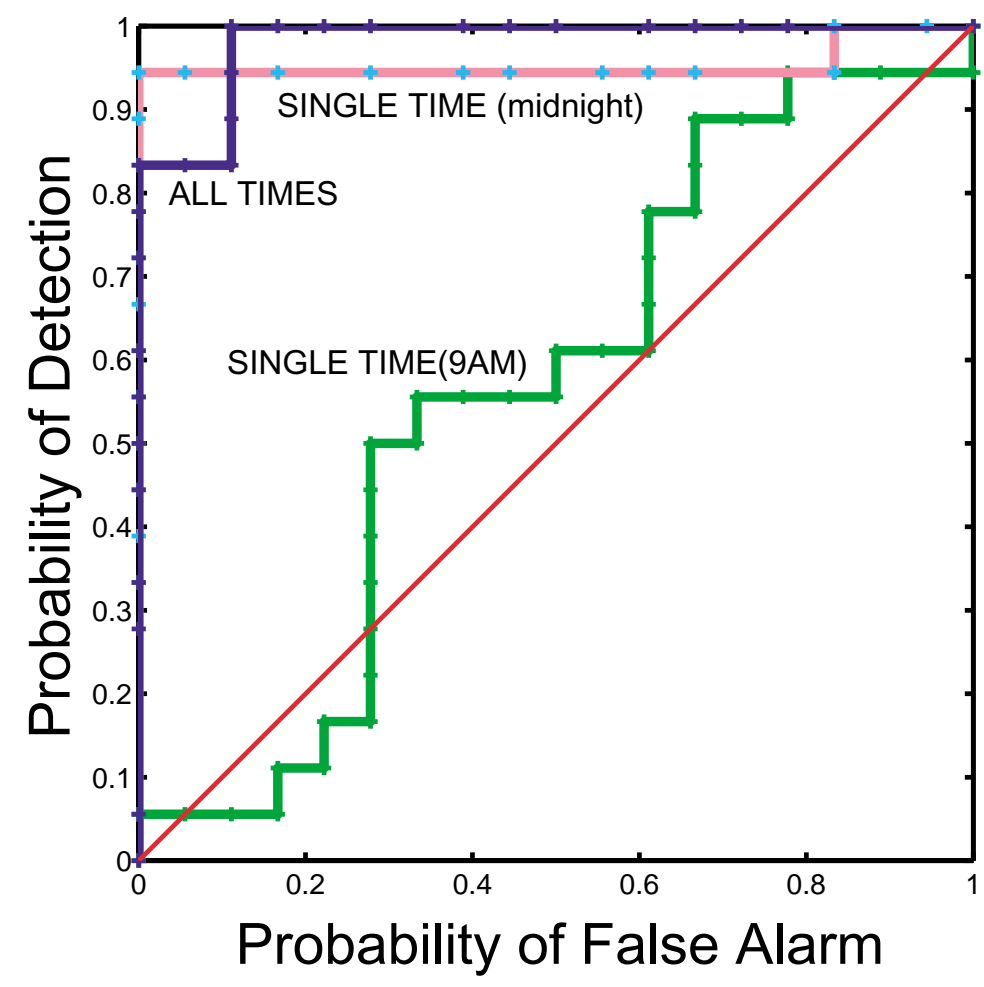

Figure 11. ROC curves comparing detection for a single time and for the full space-time history.

\section{CONCLUDING REMARKS}

We have described a number of model-based techniques for detecting buried land mines. Results from a signature simulation tool and an analysis of experimental data were used to devise a relatively simple signature model having a small number of parameters. The techniques examined include (1) the estimator-classifier technique developed by the authors, which uses model parameters and residual error via a classifier; (2) the GLRT, which uses only the residual error; (3) the RX algorithm of Reed and $\mathrm{Yu}^{4}{ }^{4}$ which assumes a signature shape; and (4) a model-free classifier of temporal samples, which ignores spatial information present in the data.

These algorithms were used to detect buried mines in imagery acquired during July 1998 at Fort A.P. Hill, VA. That data set comprises imagery from 15 mines collected at several times over a 24 hour cycle. The detection performance of these algorithms was compared. The results suggest the following:

First, model-based detection algorithms can out-perform model-free algorithms, even when the model parameters must be estimated from the data. A direct comparison of the estimator-classifier method and the GLRT, both of which use the same model, suggests that the model parameters have information that significantly improves detection.

Second, detection performance using only spatial information from a single well-chosen time, can be comparable to the performance achieved using the complete time history of the signature. The choice of sample time, however, is critical to success.

Finally, the spatial shape of mine signatures varies considerably, and it is not sufficient to use a single universal shape for all mines. The performance of the RX algorithm, which assumes a constant mine shape, was significantly inferior to even that of the temporal classifier.

\section{ACKNOWLEDGMENTS}

This project was supported in part by funds from Duke University under an award from the ARO (the OSD MURI program). The findings, opinions and recommendations expressed therein are those of the author and are not necessarily those of Duke University or the ARO. 


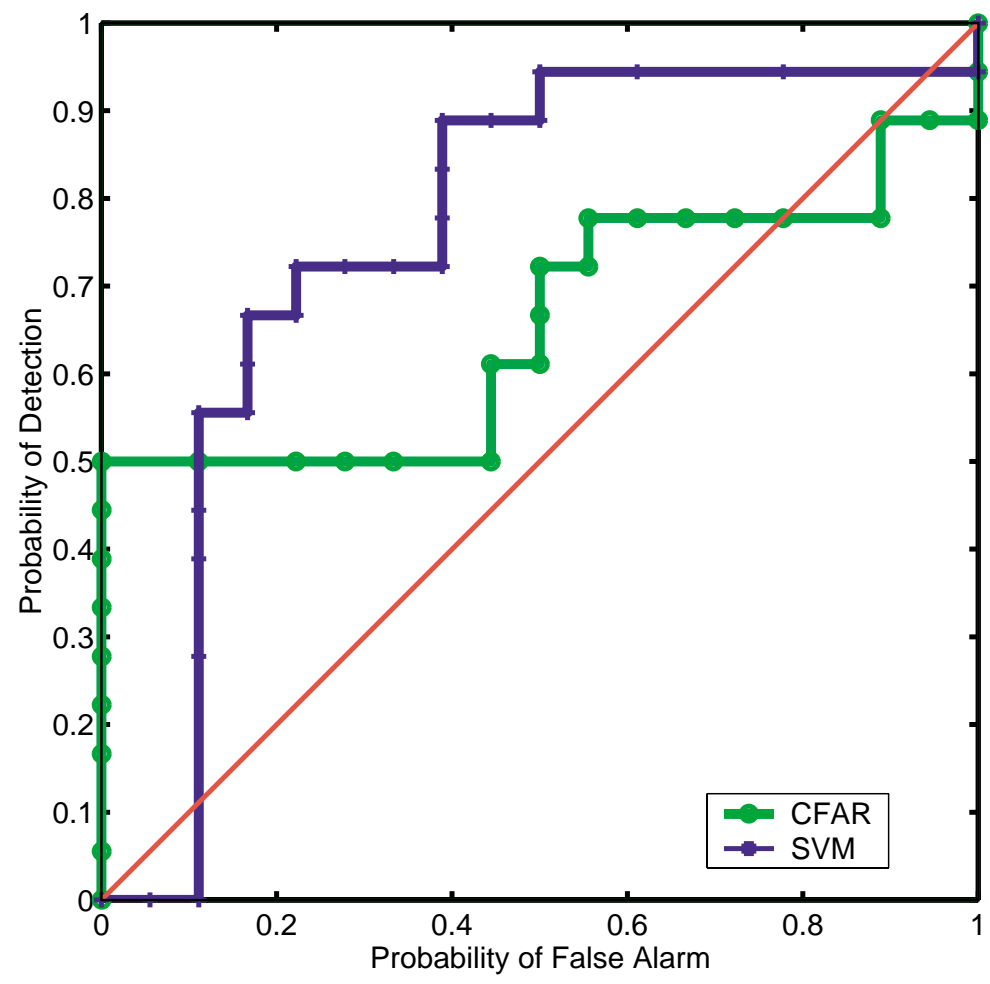

Figure 12. ROC curves for (1) a CFAR algorithm that uses spatial and temporal information with an assumed constant mine signature, and (2) a temporal-only model-free SVM classifier.

\section{REFERENCES}

1. I. K. Sendur and B. A. Baertlein, "Simulation of thermal IR buried mine signatures," in Detection and Remediation Technologies for Mines and Minelike Targets V, A. C. Dubey, J. F. Harvey, T. J. Broach, and R. E. Dugan, eds., Proceedings of SPIE 4038, pp. 156-166, 2000.

2. B. A. Baertlein and I. K. Sendur, "The role of environmental factors and mine composition in thermal IR mine signatures," in Detection and Remediation Technologies for Mines and Minelike Targets VI, A. C. Dubey, J. F. Harvey, T. J. Broach, and V. George, eds., Proceedings of SPIE 4394, p. this issue, 2001.

3. M. Lundberg and I. Y. H. Gu, "A 3-D matched filter for detection of land mines using spatio-temporal thermal modeling," in Detection Technologies for Mines and Minelike Targets V, A. C. Dubey, J. F. Harvey, J. T. Broach, and R. E. Dugan, eds., Proc. SPIE 4038, pp. 179-188, 2000.

4. I. S. Reed and X. Yu, "Adaptive multiple-band CFAR detection of an optical pattern with unknown spectral distribution," IEEE Trans. Acoustics, Speech and Signal Proc. 38, pp. 1760-1770, October 1990.

5. P. Gao and L. Collins, "Improved signal processing approaches for landmine detection," in Detection and Remediation Technologies for Mines and Minelike Targets III, A. C. Dubey, J. F. Harvey, and J. T. Broach, eds., SPIE 3392, pp. 1034-1043, 1998.

6. H. V. Poor, An Introduction ot Signal Detection and Estimation, Springer-Verlag, New York, NY, 2nd ed., 1994.

7. V. Vapnik, The Nature of the Statistical Learning Theory, Springer Verlag, New York, NY, second ed., 2000.

8. B. Schölkopf, C. J. C. Burges, and A. J. Smola, Advances in Kernel Methods: Support Vector Learning, MIT Press, Cambridge, MA, 1999. 